Full length article

\title{
Hip-Knee-Ankle (HKA) angle modification during gait in healthy subjects
}

\author{
Julien Clément ${ }^{\mathrm{a}, \mathrm{b}}$, William Blakeney ${ }^{\mathrm{a}}$, Nicola Hagemeister ${ }^{\mathrm{b}}$, François Desmeules ${ }^{\mathrm{a}}$, \\ Neila Mezghani ${ }^{\mathrm{b}, \mathrm{c}}$, Véronique Lowry ${ }^{\mathrm{a}}$, Pascal-André Vendittoli ${ }^{\mathrm{a}, *}$ \\ ${ }^{a}$ Hôpital Maisonneuve-Rosemont, Université de Montréal, 5415 Boulevard de l'Assomption, Montréal, Québec, H1T 2M4, Canada \\ ${ }^{\mathrm{b}}$ Laboratoire de Recherche en Imagerie et Orthopédie, École de Technologie Supérieure, Centre de recherche, Centre Hospitalier de l'Université de Montréal, Tour Viger, 900, \\ rue St-Denis, Local R11.322, Montréal, Québec, H2X 0A9, Canada \\ ${ }^{\mathrm{c}}$ Centre de Recherche LICEF, Université TÉLUQ, 5800, rue Saint-Denis, bureau 1105, Montréal, Québec, H2S 3L5, Canada
}

\section{A R T I C L E I N F O}

\section{Keywords:}

Healthy subjects

3D knee kinematics

Dynamic hip-knee-angle

KneeKG ${ }^{\mathrm{TM}}$ system

\begin{abstract}
A B S T R A C T
Background: Achieving a neutral static Hip-Knee-Ankle angle (sHKA) measured on radiographs has been considered a factor of success for total knee arthroplasty (TKA). However, recent studies have shown that sHKA seems to have no effect on TKA survivorship. sHKA is not representative of the dynamic loading occurring during gait, unlike the dynamic HKA (dHKA).

Research question: The primary objective was to see if the sHKA is predictive of the dynamic HKA (dHKA). A secondary objective was to document to what degree the dHKA changes during gait.

Methods: We analysed 3D knee kinematics during gait of a cohort of 90 healthy individuals with the KneeKG ${ }^{\mathrm{TM}}$ system. dHKA was calculated and compared with sHKA. Knees were considered "Stable" if the dHKA remained in valgus or varus for greater than $95 \%$ of the corresponding phase, and "Changer" otherwise. Patient characteristics of the Stable and Changer knees were compared to find associated factors.

Results: Absolute variation of dHKA during gait was $10.9 \pm 5.3^{\circ}$ for the whole cohort. The variation was less for the varus knees $\left(10.3 \pm 4.8^{\circ}\right)$, than for the valgus knees $\left(12.8 \pm 6.1^{\circ}, \mathrm{p}=0.008\right)$. We found low to moderate correlations $(r=0.266$ to $0.553, \mathrm{p}<0.001$ ) between sHKA and dHKA values for varus knees and no significant correlation for valgus knees. Twenty two percent (36/165) of the knees were considered Changers. The proportion of knees that were Changers was $15 \%$ of the varus versus $39 \%$ of the valgus ( $\mathrm{p}<0.001$ ).

Significance: Lower limb radiographic measures of coronal alignment have limited value for predicting dynamic measures of alignment during gait.
\end{abstract}

\section{Introduction}

Hip-Knee-Ankle angle (HKA) measured on standing full-length radiographs is a common evaluation method to assess lower limb anatomy, diagnose pathologies, as a surgical planning tool and to assess success of the surgical procedure. A neutral coronal HKA has been the key alignment goal for total knee arthroplasty (TKA) for many years. It was believed to improve TKA survivorship and polyethylene wear. However, several long-term studies have recently failed to find a significant relationship between HKA and TKA survivorship [1-4]. These results could be explained by the fact that static HKA (sHKA) may be of limited value in estimating the dynamic loading that occurs during gait.

Dynamic measures have thus been gaining popularity in recent years. It has been shown that the healthy knee adduction angle through stance is correlated with the dynamic load on the medial compartment of the knee [5-7], with a greater angle resulting in a larger adduction moment and increased load. Increased knee adduction moments have also been reported in patients with osteoarthritis (OA) compared to healthy controls [8-10], while increased dynamic loading of the knee joint has been associated with the disease progression [11]. Finally, a larger knee adductor moment has been associated with early loosening of TKA prostheses [12,13].

Dynamic angular measurements of the knee seem to be a key factor of the progression of knee OA and the survivorship of TKA. The concept of the dynamic HKA (dHKA), which measures the coronal alignment of the knee throughout the gait cycle, has thus been considered as an alternative measure to the static sHKA. The dHKA may more accurately reflect the loading that occurs in the knee, and therefore be more predictive of long-term outcomes. To our knowledge, only one study [14] compared the sHKA with the dHKA during gait, in a healthy population. Duffell et al. recruited nine healthy participants, and found the dHKA to be in significantly greater varus during gait (by a mean of

\footnotetext{
* Corresponding author at: Hôpital Maisonneuve-Rosemont, Service d'orthopédie, 5415 Boul L'Assomption, Montréal, QC, H1T 2M4, Canada.

E-mail address: pa.vendittoli@me.com (P.-A. Vendittoli).
} 
Table 1

Mean demographic and radiographic data: comparison between men and women.

\begin{tabular}{|c|c|c|c|c|}
\hline & Entire cohort $(\mathrm{n}=90$ subjects, $\mathrm{n}=165$ knees $)$ & Women ( $\mathrm{n}=49$ subjects, $\mathrm{n}=90$ knees) & Men ( $\mathrm{n}=41$ subjects, $\mathrm{n}=75$ knees $)$ & p-value \\
\hline Age (years) & $34.8(\min 20.0, \max 64.0, \mathrm{SD} 12.3)$ & $34.8(\min 20.0, \max 63.0, \mathrm{SD} 12.7)$ & $34.8(\min 21.0, \max 64.0, \mathrm{SD} 12.1)$ & 0.997 \\
\hline Height (m) & $1.69(\min 1.42, \max 1.91, \mathrm{SD} 0.11)$ & $1.62(\min 1.42, \max 1.78, S D: 0.08)$ & $1.78(\min 1.62, \max 1.91, \mathrm{SD}: 0.06)$ & $<0.001$ \\
\hline Mass (kg) & $71.8(\min 36.0, \max 118.0$, SD 17.3$)$ & $62.5(\min 36.0, \max 111.0$, SD 14.4$)$ & $83.0(\min 63.0, \max 118.0, \mathrm{SD} 13.5)$ & $<0.001$ \\
\hline $\mathrm{BMI}\left(\mathrm{kg} / \mathrm{m}^{2}\right)$ & $24.8(\min 17.1, \max 40.8 \mathrm{SD} 4.6)$ & $23.7(\min 17.1, \max 40.8, \mathrm{SD} 4.7)$ & $26.1(\min 19.4, \max 36.9, \operatorname{SD} 4.1)$ & 0.012 \\
\hline Walking speed $(\mathrm{m} / \mathrm{s})$ & $0.76(\min 0.56, \max 1.08, \text { SD } 0.11)^{*}$ & $0.75(\min 0.56, \max 1.03, \mathrm{SD} 0.12)$ & $0.78(\min 0.56, \max 1.08, \mathrm{SD} 0.11)^{1 . k}$ & 0.299 \\
\hline mLDFA $\left({ }^{\circ}\right)$ & $88.3(\min 82.2, \max 93.6, \mathrm{SD} 2.3)$ & $88.5(\min 82.3, \max 93.4, \mathrm{SD} 2.3)$ & $88.1(\min 82.2, \max 93.6, \mathrm{SD} 2.4)$ & 0.252 \\
\hline $\operatorname{aLDFA}\left({ }^{\circ}\right)$ & $82.6(\min 76.3, \max 88.1, S D 2.2)$ & $82.7(\min 77.5, \max 87.8, S D 2.2)$ & $82.4(\min 76.3, \max 88.1, \mathrm{SD} 2.3)$ & 0.360 \\
\hline MPTA $\left({ }^{\circ}\right)$ & $87.5(\min 80.2, \max 92.2, \mathrm{SD} 2.4)$ & $88.4(\min 83.6, \max 92.2, \mathrm{SD} 1.7)$ & $86.4(\min 80.2, \max 91.5, \mathrm{SD} 2.6)$ & $<0.001$ \\
\hline sHKA $\left(^{\circ}\right)$ & $-1.6(\min -9.4, \max 7.3$, SD 3.0$)$ & $-0.8(\min -7.4, \max 7.3, \mathrm{SD} 2.6)$ & $-2.6(\min -9.4, \max 5.0$, SD 3.1$)$ & $<0.001$ \\
\hline
\end{tabular}

mLDFA $=$ mechanical lateral distal femoral angle.

aLDFA = anatomical lateral distal femoral angle.

MPTA $=$ mechanical medial proximal tibial angle.

sHKA $=$ static hip-knee-ankle angle.

$* \mathrm{n}=89$.

$* * \mathrm{n}=40$.

$3.5^{\circ}$ ) compared to the sHKA.

The goal of the present study was to analyse dHKA throughout the gait cycle on a healthy population and compare it with sHKA. The primary aim of the study was to see if the sHKA is predictive of the dHKA, and the secondary aim was to document to what degree the dHKA changes throughout gait. We hypothesised that the sHKA value has limited value for predicting dHKA, and that the dHKA can vary considerably during gait.

\section{Material and methods}

Ethical approval was obtained from the hospital research and ethics committee, and all participants gave informed consent. The subjects came from our recent study [15] analysing the knee kinematics of a cohort of 90 healthy individuals (49 females and 41 males). The subjects had to be 18 to 65 years old and were excluded if they had any lower limb pathology, musculoskeletal disorder, or previous lower limb surgery. Each subject had a full-length weight-bearing (FLWB) radiograph to confirm the absence of knee joint degeneration. All demographic and radiographic data are presented in Table 1.

The 3D knee kinematic analyses were performed during gait with the KneeKG ${ }^{\mathrm{TM}}$ (Emovi Inc., Montréal, QC, Canada), which is a valid and reliable tool for measuring the knee joint movements [16-21]. After the installation of the KneeKG ${ }^{\mathrm{Tm}}$ on the subjects and the calibration procedure $[17,18]$, subjects were asked to walk on a treadmill to determine their self-selected walking speed (had to be $>2 \mathrm{~km} / \mathrm{h}$ ) [22]. All kinematic data were collected by one trained technician. A complete description of the methodology can be found in our previous study [15].

\subsection{Kinematics data processing}

3D kinematic analyses of 165 knees (75 subjects had bilateral evaluations, and 15 subjects had unilateral evaluation). Kinematic data were used to generate knee angles with the Knee3D ${ }^{\mathrm{TM}}$ software (Emovi Inc.): knee flexion-extension, abduction-adduction, and internal-external rotation, representing the motion of the tibia relative to the femur [23]. Mean knee angles were computed by averaging the 15 most repeatable gait cycles in each subject [24]. The knee angle curves were normalized from 1 to $100 \%$ of the average gait cycle. dHKA was calculated by projecting the hip, knee and ankle joint centers into the subjects' frontal plane, and by measuring the angle formed by the vectors connecting the knee center to the hip and ankle centers (Fig. 1A). The joint centers were estimated during the functional calibration procedure of the $\mathrm{KneeKG}^{\mathrm{TM}}$ : $[17,18]$ the hip joint center was defined during a lower limb circumduction movement; the knee joint center was defined as the projection of the femoral epicondyle's midpoint on the functional knee flexion-extension axis and the ankle joint center
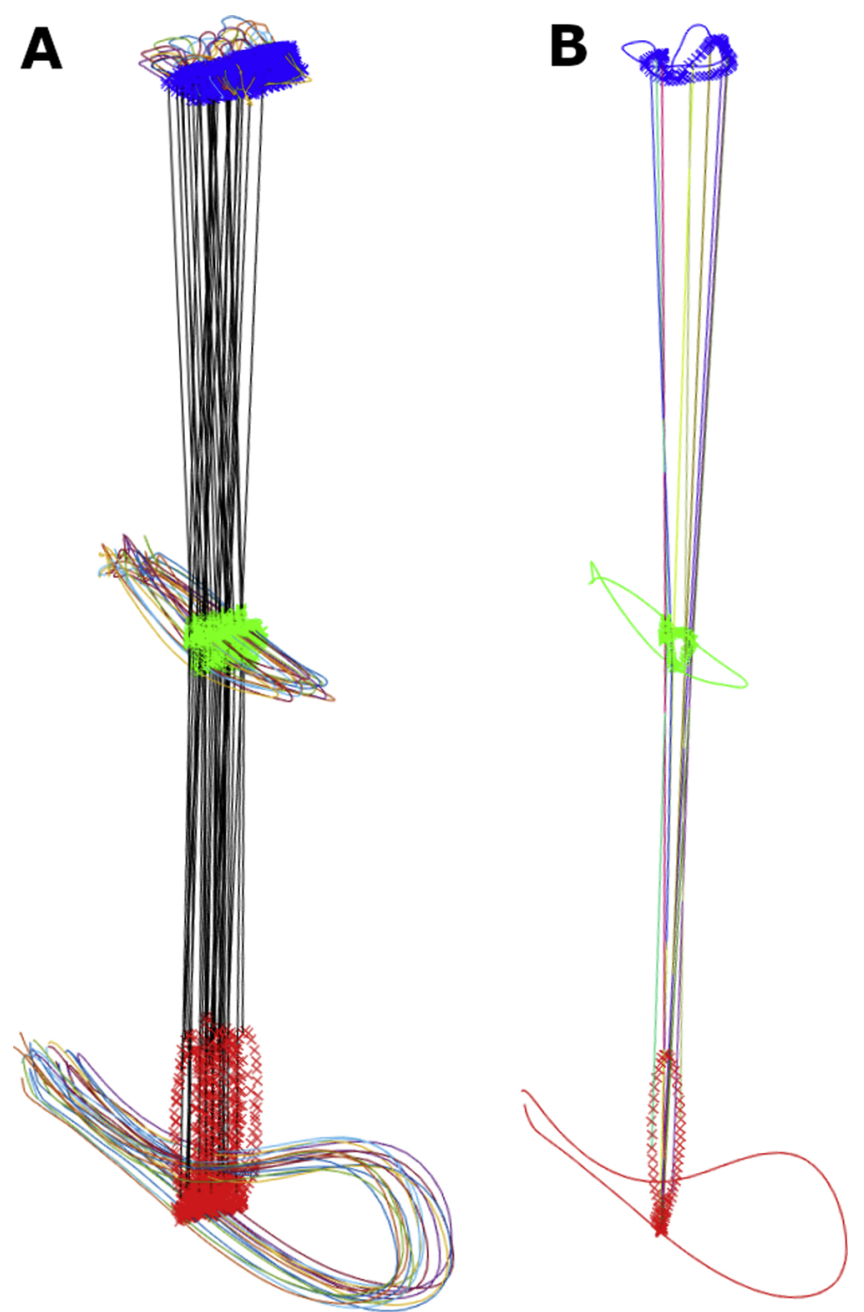

Fig. 1. (A) The 3D curves represent the movements of the hip, knee and ankle joint centers for all the gait cycles recorded on a healthy subject performing a walk of $45 \mathrm{~s}$ on a treadmill. The blue, green, and red crosses are the projections of these hip, knee and ankle curves into the subjects' frontal plane. The black lines caracterise the vectors connecting the knee center to the hip and ankle centers, and were used to measure the dHKA. (B) Represents the same things as A, but only for the 15 most repeatable gait cycles (For interpretation of the references to colour in this figure legend, the reader is referred to the web version of this article). 


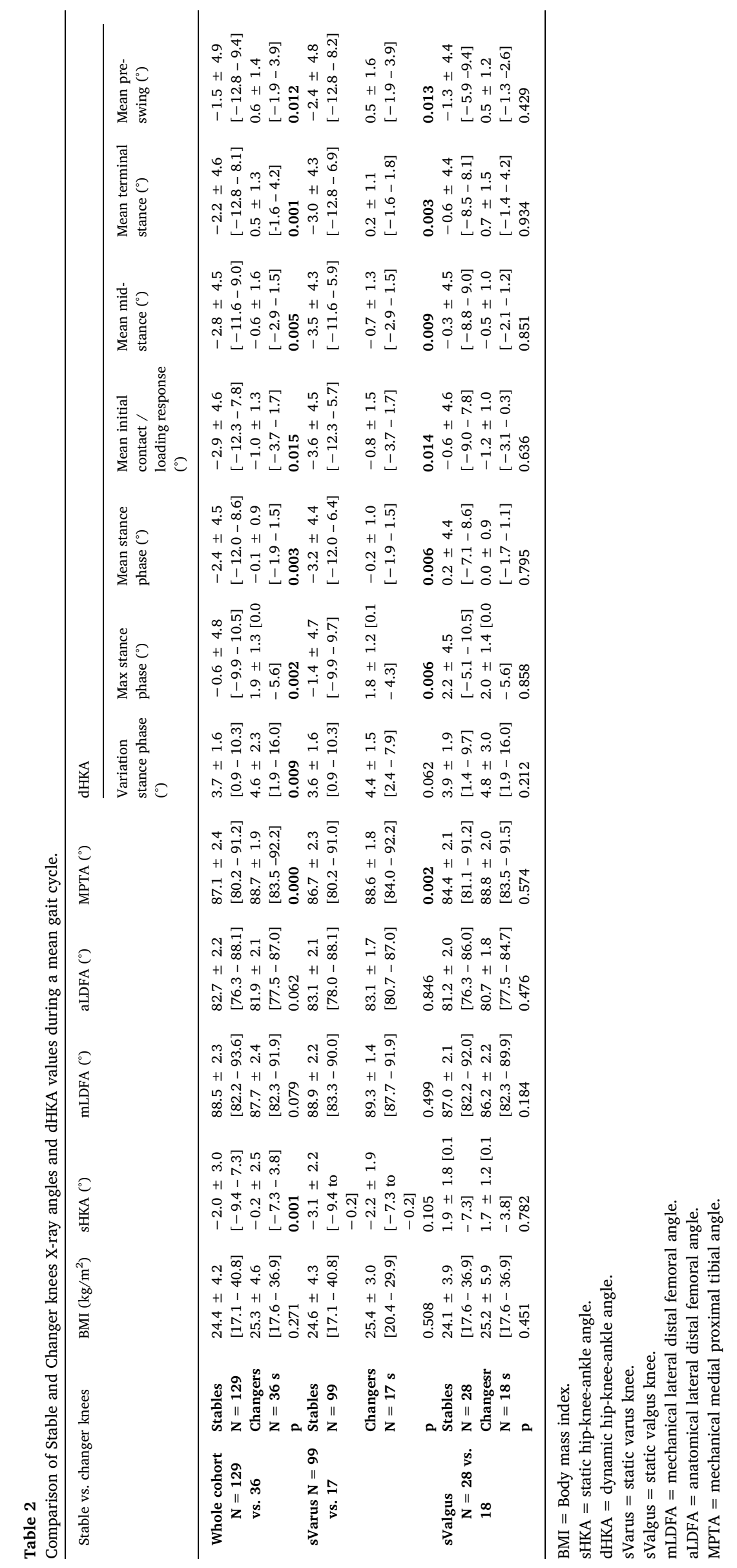


Table 3

Pearson correlation coefficients between sHKA and dHKA values for the whole cohort, the sVarus/sValgus knees group, and Stable/Changer knees group.

\begin{tabular}{|c|c|c|c|c|c|c|c|c|c|c|c|}
\hline \multirow[t]{2}{*}{ sHKA } & & \multirow{2}{*}{$\begin{array}{l}\mathrm{BMI} \\
\left(\mathrm{kg} / \mathrm{m}^{2}\right)\end{array}$} & \multirow[t]{2}{*}{$\operatorname{mLDFA}\left({ }^{\circ}\right)$} & \multirow[t]{2}{*}{$\operatorname{aLDFA}\left({ }^{\circ}\right)$} & \multirow[t]{2}{*}{$\operatorname{MPTA}\left({ }^{\circ}\right)$} & \multicolumn{6}{|l|}{ dHKA } \\
\hline & & & & & & $\begin{array}{l}\text { Max stance } \\
\text { phase }\left({ }^{\circ}\right)\end{array}$ & $\begin{array}{l}\text { Mean stance } \\
\text { phase }\left({ }^{\circ}\right)\end{array}$ & $\begin{array}{l}\text { Mean initial contact } \\
\text { / loading response } \\
\left({ }^{\circ}\right)\end{array}$ & $\begin{array}{l}\text { Mean mid- } \\
\text { stance }\left({ }^{\circ}\right)\end{array}$ & $\begin{array}{l}\text { Mean terminal } \\
\text { stance }\left({ }^{\circ}\right)\end{array}$ & $\begin{array}{l}\text { Mean pre- } \\
\text { swing }\left({ }^{\circ}\right)\end{array}$ \\
\hline Whole cohort & Pearson & 0.004 & -0.470 & -0.478 & 0.505 & 0.531 & 0.517 & 0.460 & 0.478 & 0.551 & 0.523 \\
\hline $\mathrm{N}=165$ & Sign. & 0.963 & 0.000 & 0.000 & 0.000 & 0.000 & 0.000 & 0.000 & 0.000 & 0.000 & 0.000 \\
\hline \multirow[t]{2}{*}{ sVarus $N=116$} & Pearson & -0.109 & -0.316 & -0.321 & 0.556 & 0.531 & 0.519 & 0.495 & 0.482 & 0.532 & 0.534 \\
\hline & Sign. & 0.245 & 0.001 & 0.000 & 0.000 & 0.000 & 0.000 & 0.000 & 0.000 & 0.000 & 0.000 \\
\hline \multirow[t]{2}{*}{ sValgus $N=46$} & Pearson & 0.481 & -0.081 & -0.057 & -0.020 & 0.210 & 0.114 & 0.099 & 0.043 & 0.176 & 0.136 \\
\hline & Sign. & 0.001 & 0.594 & 0.707 & 0.896 & 0.161 & 0.450 & 0.515 & 0.778 & 0.243 & 0.367 \\
\hline Stable stance & Pearson & -0.051 & -0.392 & -0.412 & 0.514 & 0.539 & 0.529 & 0.489 & 0.491 & 0.553 & 0.548 \\
\hline$N=129$ & Sign. & 0.565 & 0.000 & 0.000 & 0.000 & 0.000 & 0.000 & 0.000 & 0.000 & 0.000 & 0.000 \\
\hline Changer stance & Pearson & 0.109 & -0.721 & -0.692 & 0.210 & 0.213 & 0.113 & -0.176 & 0.015 & 0.294 & 0.043 \\
\hline $\mathbf{N}=36$ & Sign. & 0.527 & 0.000 & 0.000 & 0.220 & 0.211 & 0.510 & 0.304 & 0.933 & 0.082 & 0.803 \\
\hline
\end{tabular}

$\mathrm{BMI}=$ Body mass index.

sHKA = static hip-knee-ankle angle.

dHKA = dynamic hip-knee-ankle angle.

sVarus = static varus knee.

sValgus $=$ static valgus knee.

mLDFA = mechanical lateral distal femoral angle.

aLDFA = anatomical lateral distal femoral angle.

MPTA $=$ mechanical medial proximal tibial angle.

was defined as the midpoint of the malleoli. Mean changes in the dHKA during walking were also determined by averaging the 15 most repeatable gait cycles (Fig. 1-B). Maximum and mean dHKA for the gait cycle, stance and swing phases, as well as mean dHKA for initial contact/loading response, mid-stance, terminal stance, and pre-swing phases were calculated. Knees were considered "Stable" if the dHKA remained positive or negative - i.e. in valgus or varus - for greater than $95 \%$ of the corresponding phase and were considered "Changer" otherwise.

\subsection{Radiographic data collection}

On FLWB X-rays: mechanical and anatomical lateral distal femoral angle (mLDFA and aLDFA), mechanical medial proximal tibial angle (MPTA), and static hip-knee-ankle angle (sHKA) were measured by one investigator with Impax software (Agfa Healthcare, Mortsel, Belgium) $[15,25]$.

\subsection{Statistical analyses}

Descriptive statistics of both "Stable" and "Changer" knees were computed and compared to the sHKA values. Pearson correlation coefficients between sHKA and dHKA values were analysed for the whole cohort, for the static varus (sVarus) / static valgus (sValgus) knees, and for the Stable and Changer knees separately. The strength of the correlations were classified as: $0.9-1.0$ for very high correlation; 0.7-0.9 for high correlation; 0.5-0.7 for moderate correlation; $0.3-0.5$ for low correlation and; $0.0-0.3$ for negligible correlation [26]. Stable and Changer knees were compared according to their dHKA and sHKA using the Student t-test. The same comparisons were performed for male and female demographic, radiographic and kinematic data. Significance was set at alpha of 0.05 . All data processing and statistical analyses were undertaken with MATLAB R2018a (Natick, MA, USA) and SPSS 24.0 (IBM Corp., Armonk, NY, USA), respectively.

\section{Results}

Among the 165 knees analysed in the present study, 116 (70\%) were in sVarus (sHKA < 0), $46(28 \%)$ in sValgus (sHKA > 0) and $3(2 \%)$ in static neutral $(\mathrm{sHKA}=0)$. dHKA absolute variation was $10.9 \pm 5.3^{\circ}$ $\left[2.4^{\circ}-28.3^{\circ}\right]$ for the whole cohort and less for the sVarus knees $\left(10.3 \pm 4.8^{\circ}\left[2.4^{\circ}-26.3^{\circ}\right]\right)$, than for the sValgus knees $\left(12.8 \pm 6.1^{\circ}\right.$ $\left.\left[2.9^{\circ}-28.3^{\circ}\right], \mathrm{p}=0.008\right)$. Larger variations were present during the swing phase: $9.7 \pm 4.9^{\circ}\left[1.4^{\circ}-25.5^{\circ}\right]$ for the whole cohort; $9.3 \pm 4.7^{\circ}$ $\left[1.4^{\circ}-24.8^{\circ}\right]$ for the sVarus knees and; $11.1 \pm 5.4^{\circ}\left[2.2^{\circ}-25.5^{\circ}\right]$ for the sValgus knees (more than sVarus knees, $\mathrm{p}=0.032$ ). dHKA absolute variations during the stance phase were: $3.9 \pm 1.8^{\circ}\left[0.9^{\circ}-16.0^{\circ}\right]$ for the whole cohort; $3.7 \pm 1.6^{\circ}\left[0.9^{\circ}-10.3^{\circ}\right]$ for the sVarus knees and; $4.2 \pm 2.4^{\circ}\left[1.4^{\circ}-16.0^{\circ}\right]$ for the sValgus knees $(p=0.144)$. Comparisons between Stable and Changer knees, and correlations between sHKA and dHKA, were then computed for the stance phase.

There were 129 of 165 knees (78\%) that were considered as Stable, and $36(22 \%)$ as Changers. Of the 129 Stables knees, 87 (67\%) were in dVarus versus $42(33 \%)$ in dValgus ( $p<0.001)$. There were 99 Stable knees in sVarus ( $85 \%$ of sVarus) versus 28 in sValgus ( $61 \%$ of sValgus) ( $\mathrm{p}<0.001)$, and 2 Stable knees were in static neutral $(67 \%$ of neutral). Of the 36 Changer knees, 17 were in sVarus (15\% of sVarus) and 18 were in sValgus (39\% of sValgus) ( $\mathrm{p}<0.001$ ), while 1 was in static neutral ( $33 \%$ of neutral). On average, the Changer knees were in dVarus for $55 \pm 25 \%$ of the stance phase, and $45 \pm 25 \%$ for dValgus. In subjects where both knees were tested $(n=75), 7$ had bilateral Changer knees, 18 had one Changer and one Stable knees, and 50 had bilateral Stable knees.

The dHKA absolute variation for the Stable knees was $3.7^{\circ} \pm 1.6^{\circ}$ $\left[0.9^{\circ}-10.3^{\circ}\right]$, while their sHKA was $-2.0^{\circ} \pm 3.0^{\circ}\left[-9.4^{\circ}-7.3^{\circ}\right]$ (p < 0.001). Among the 129 Stable knees, $53(41 \%)$ had an amplitude of dHKA inferior to $3^{\circ}$, and $76(59 \%)$ superior to $3^{\circ}$. The amplitude of dHKA for the Changer knees was $4.6^{\circ} \pm 2.3^{\circ}\left[1.9^{\circ}-16.0^{\circ}\right]$ (higher than Stable knees, $\mathrm{p}=0.009)$, while their sHKA was $-0.2^{\circ} \pm 2.5^{\circ}\left[-7.3^{\circ}-\right.$ $3.8^{\circ}$ ] (more in valgus than Stable knees, $\mathrm{p}=0.001$ ) (Table 2). Among the 36 Changer knees, $7(19 \%)$ had an amplitude of dHKA inferior to $3^{\circ}$, and $29(81 \%)$ superior to $3^{\circ}$.

\subsection{Correlations between sHKA vs. dHKA}

Table 3 shows the Pearson correlation coefficients between sHKA and DHKA values for the whole cohort and the various groups (i.e. sVarus/sValgus knees, and Stable/Changer knees). There were significant correlations with low to moderate coefficients ( $r=0.266$ to 0.553, $\mathrm{p}<0.001$ ) between sHKA and the dHKA values for the whole cohort, the sVarus knees (Fig. 2-A), and the Stable knees groups. Conversely, there was no significant correlation between sHKA and the 

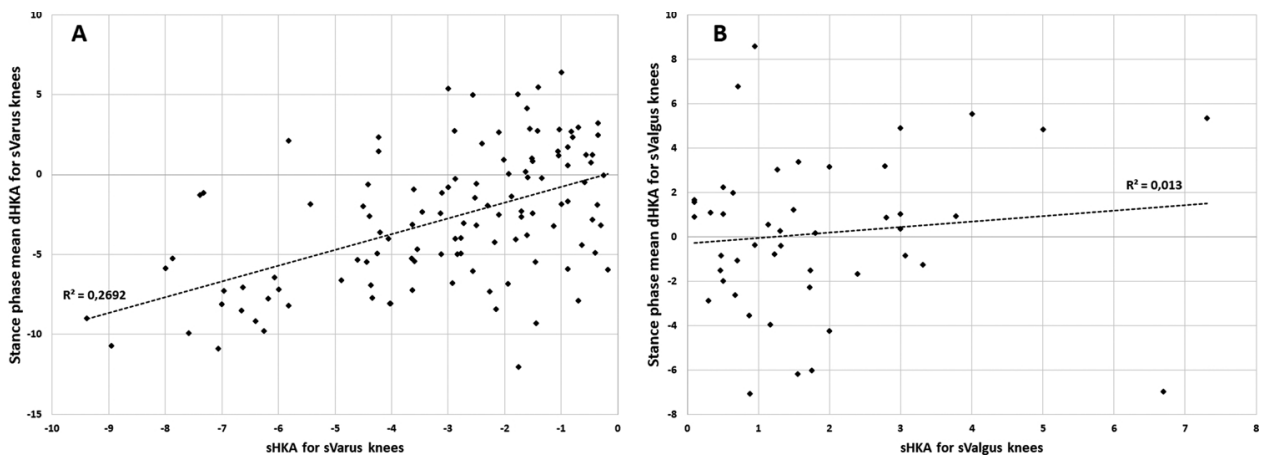

Fig. 2. Correlation between sHKA and means values of dHKA during the stance phase for the sVarus (A) and sValgus knees (B).

dHKA values for the sValgus knees (Fig. 2-B) and the Changer knees groups.

\subsection{Comparison between stable and changer knees}

When comparing Stable and Changer knees, significant differences were found for sHKA $\left(-2.0^{\circ}\right.$ vs. $\left.-0.2^{\circ}, \mathrm{p}=0.001\right)$, MPTA $\left(87.1^{\circ}\right.$ vs. $\left.88.7^{\circ}, \mathrm{p}<0.001\right)$, amplitude of dHKA (3.7 $7^{\circ}$ vs. $\left.4.6^{\circ}, \mathrm{p}=0.009\right)$, and all other dHKA values ( $\mathrm{p}<0.005)$ (Table 2). For the knees in sVarus, Stable knees had smaller MPTA $\left(86.7^{\circ}\right.$ vs. $\left.88.6^{\circ}, \mathrm{p}=0.002\right)$ and smaller dHKA values $(\mathrm{p}<0.005)$ than Changer knees. Conversely, no significant differences were found between Stable and Changer knees in sValgus. Comparing men and women results (Table 4), all dHKA values were significantly smaller in men than in women $(p<0.001)$, except for the absolute variation during the stance phase.

\section{Discussion}

The coronal alignment of the knee measured on a FLWB radiograph (sHKA) is still one of the key measures for clinicians performing a mechanically aligned TKA. However, the usefulness of this measure in predicting implant survivorship has been questioned. This may be explained by the poor correlation between the sHKA and the dynamic coronal alignment of the knee (dHKA) during gait, which has proven to be a good indicator of the dynamic load in the knee. On the other hand, the kinematically aligned TKA aims at restoring dHKA throughout gait cycle, but a clear description of normal dHKA on a large healthy cohort is still lacking. The main objective of the present study was to describe the dHKA of healthy subjects and see if it is correlated with sHKA. We found a low to moderate correlation $(r=0.266$ to $0.553, p<0.001)$ between sHKA and the dHKA values for sVarus knees and no correlation for sValgus knees. Furthermore, a number of the knees $(22 \%, 36 / 165)$ demonstrated a switch in the HKA from dVarus to dValgus (or viceversa) during at least $5 \%$ of the stance phase of gait. These results underscore the limited value of radiographic measures of coronal alignment for predicting dynamic measures of alignment during gait. One of the main reasons for the differences between sHKA and dHKA could be the torsion of the femur, which cannot be quantified on FLWB radiograph. Indeed, during knee flexion, the tibia rotates around the femoral condyles. The orientation of the flexion-extension axis, passing through the femoral condyles, directly influences the dHKA.

The magnitude of the changes in the dHKA were a mean of $10.9^{\circ}$ (range $2.4^{\circ}-28.3^{\circ}$ ). This large amplitude mainly occurred during the swing phase, with a mean variation of $9.7^{\circ}$ (range $1.4^{\circ}-25.5^{\circ}$ ), versus the stance where a mean of $3.9^{\circ}$ (range $0.9^{\circ}-16.0^{\circ}$ ) was observed. This difference may be explained by the larger knee range of motion during swing phase compared to stance phase. With this great variation in the dHKA throughout gait, one may question the value of a single static measurement of alignment for pre-operative planning and as a postoperative measure of success. Moreover, in $22 \%$ of the knees, a switch between varus and valgus was present. On average, these Changer knees were in dVarus for $55 \%$ of the stance phase, and in dValgus for $45 \%$ of the stance phase. In proportion, more Changer knees were in sValgus than in sVarus (39\% of sValgus versus $15 \%$ of sValgus, $\mathrm{p}<0.001)$. Knees in sValgus are thus more likely to be Changer than sVarus knees.

We found only one other study comparing sHKA with dHKA in a cohort of healthy knees. Duffell et al. looked at 9 healthy participants and reported a significant change $(\mathrm{p}<0.01)$ from a mean sHKA of $0.5^{\circ}$ valgus (SD 2.8) to a mean dHKA of $4.4^{\circ}$ varus (SD 3.0). [14] Again the greatest change was seen in the valgus knees, that went from a mean of $2.0^{\circ}$ valgus (SD 1.2) statically to a mean of $3.8^{\circ}$ varus (SD 1.9, $\mathrm{p}<0.01$ ) during gait. This study used motion capture tracking system to calculate both the dHKA and the sHKA, but no radiographs were taken.

In TKA knees, Rivière et al. in a cohort of 35 patients, using motioncapture gait analysis, found no significant correlation between the sHKA and the dHKA during stance phase $(r=0.14, p=0.45)$ [27]. They found sHKA to have a moderate correlation with the mean and peak adduction moments $(r=0.31$ and $r=-0.352$ respectively). Similarly, Orishimo et al. performing gait analysis on a cohort of 15 patients before and after TKA surgery, did not find any correlation between standing or dynamic coronal alignment of TKAs with peak adduction moments [28]. Moreover, improvement in the sHKA to neutral post-surgery did not correlate with a change in the peak adduction moments during gait. Miller et al. looked at the plateau load distributions in 15 patients post-TKA. The TKAs were neutrally aligned on post-operative radiographs and produced balanced loading between the tibial plateaus in the standing position [29]. However, less than half of the knees ( $40 \%$ at 2 years) achieved equal plateau load distributions in dynamic loading. In fact, both pre-operatively and post-operatively the dynamic load was independent of the sHKA.

It thus appear that sHKA poorly reflects dHKA, and therefore knee loading. That may explain why the sHKA, which is used to perform mechanically aligned TKA, has shown to be of poor value to predict implant survivorship [1-4]. As an alternative of mechanical alignment, kinematic alignment is a new technique for TKA to restore native femoro-tibial joint line orientation and laxity throughout knee range of motion [30]. However, precise indications for kinematically aligned TKA remain to be defined. There is currently a trend to not reproduce severe frontal constitutional deformity for fear of overloading the TKA, and therefore increase risk of failure. The description of a normal range of dHKA in a healthy population may help to define the limits of a kinematic aligned TKA.

There are no doubt other factors apart from coronal prosthetic alignment that affect how the knee will be loaded dynamically. A study looked at the joint line orientation in both healthy and osteoarthritic subjects [31]. It demonstrated that despite a range of sHKA alignments, the joint line remained parallel to the ground when standing in the healthy subjects. Conversely, in the subjects with OA, the joint line tended to slope down medially. Interestingly, studies of kinematic alignment for TKA have also demonstrated that the post-operative joint 
line tends to be parallel to the ground, whereas it tends to slope down laterally in mechanically aligned TKAs [32,33]. The resultant functional joint line orientation may well be favourable for the overall load profile of a prosthetic joint.

Our study has some limitations. Soft tissue artefact may be an issue with traditional skin markers in gait studies. The KneeKG system was developed to overcome this with the use of its harness and exoskeleton attachment system. The accuracy and reproducibility have been assessed, with a mean accuracy of $0.4^{\circ}$ to $0.8^{\circ}$ for measured angles $[18,20]$. On the other hand, the exoskeleton may not fit on all patients anatomies and some patients may have been excluded based on their extreme anatomy (morbidly obese, very short/tall person, etc.). Collected data are also limited to only one aspect of patient gait (walking at comfortable speed $2-4 \mathrm{~km} / \mathrm{h}$ ). We cannot extrapolate the dHKA during running or jumping for example. It is important to note that the dHKA is not always well-defined in gait studies. Many studies simply measure the abduction/adduction angle between the tibia and the femur in a 3dimensional space. In our method, dHKA was calculated in the 2-dimensional frontal plane, allowing appropriate comparison with radiographs.

\section{Conclusions}

This study involving healthy subjects showed that there was only a mild to moderate correlation between sHKA and the dHKA for varus knees, and no correlation between the sHKA and dHKA for valgus knees. Furthermore, a number of the knees demonstrated a switch in the dHKA from varus to valgus, or vice-versa. Radiographic measures of coronal alignment have limited value for predicting dynamic measures of alignment during gait in a healthy population.

\section{Conflicts of interest}

The authors, their immediate families and any research foundations with which they are affiliated have not received any financial payments or other benefits from any commercial entity related to the subject of this article.

\section{Acknowledgment}

We gratefully acknowledge the technical assistance of Serge Marquis in this work.

\section{References}

[1] S. Parratte, M.W. Pagnano, R.T. Trousdale, D.J. Berry, Effect of postoperative mechanical axis alignment on the fifteen-year survival of modern, cemented total knee replacements, J. Bone Joint Surg. Am. 92 (2010) 2143-2149.

[2] T.J. Bonner, W.G.P. Eardley, P. Patterson, P.J. Gregg, The effect of post-operative mechanical axis alignment on the survival of primary total knee replacements after a follow-up of 15 years, J. Bone Joint Surg. Br. 93 (2011) 1217-1222.

[3] S.S. Morgan, A. Bonshahi, N. Pradhan, A. Gregory, A. Gambhir, M.L. Porter, The influence of postoperative coronal alignment on revision surgery in total knee arthroplasty, Int. Orthop. 32 (2008) 639-642.

[4] M.P. Abdel, M. Ollivier, S. Parratte, R.T. Trousdale, D.J. Berry, M.W. Pagnano, Effect of postoperative mechanical Axis alignment on survival and functional outcomes of modern total knee arthroplasties with cement: a concise follow-up at 20 years, J. Bone Joint Surg. Am. 100 (2018) 472-478.

[5] T.A. Gerbrands, M.F. Pisters, B. Vanwanseele, Individual selection of gait retraining strategies is essential to optimally reduce medial knee load during gait, Clin. Biomech. 29 (2014) 828-834.

[6] T. Maneekittichot, P. Sorachaimetha, P. Onmanee, T. Chanthasopeephan, The effect of vary varus malalignment on knee adduction moment during walking of human normal gait, 2013 35th Annual International Conference of the IEEE Engineering in Medicine and Biology Society (EMBC), (2013), pp. 7229-7232.

[7] H. Marouane, A. Shirazi-Adl, Sensitivity of medial-lateral load sharing to changes in adduction moments or angles in an asymptomatic knee joint model during gait, Gait Posture 70 (2019) 39-47.

[8] L. Sharma, D.E. Hurwitz, E.J. Thonar, J.A. Sum, M.E. Lenz, D.D. Dunlop, et al., Knee adduction moment, serum hyaluronan level, and disease severity in medial tibiofemoral osteoarthritis, Arthritis Rheum. 41 (1998) 1233-1240.

[9] N. Foroughi, R. Smith, B. Vanwanseele, The association of external knee adduction 
moment with biomechanical variables in osteoarthritis: a systematic review, Knee 16 (2009) 303-309.

[10] N. Foroughi, R.M. Smith, A.K. Lange, M.K. Baker, M.A. Fiatarone Singh, B. Vanwanseele, Dynamic alignment and its association with knee adduction moment in medial knee osteoarthritis, Knee 17 (2010) 210-216.

[11] T. Miyazaki, M. Wada, H. Kawahara, M. Sato, H. Baba, S. Shimada, Dynamic load at baseline can predict radiographic disease progression in medial compartment knee osteoarthritis, Ann. Rheum. Dis. 61 (2002) 617-622.

[12] T.P. Andriacchi, T.S. Stanwyck, J.O. Galante, Knee biomechanics and total knee replacement, J. Arthroplasty 1 (1986) 211-219.

[13] A. Kagan 2nd, Mechanical causes of loosening in knee joint replacement, J. Biomech. 10 (1977) 387-391.

[14] L.D. Duffell, J. Mushtaq, M. Masjedi, J.P. Cobb, The knee adduction angle of the osteo-arthritic knee: a comparison of 3D supine, static and dynamic alignment, Knee 21 (2014) 1096-1100.

[15] J. Clément, P. Toliopoulos, N. Hagemeister, F. Desmeules, A. Fuentes, P. A. Vendittoli, Healthy 3D knee kinematics during gait: differences between women and men, and correlation with x-ray alignment, Gait Posture 64 (2018) 198-204.

[16] J. Clément, J.A. de Guise, A. Fuentes, N. Hagemeister, Comparison of soft tissue artifact and its effects on knee kinematics between non-obese and obese subjects performing a squatting activity recorded using an exoskeleton, Gait Posture 91 (2018) 197-203.

[17] N. Hagemeister, L.H. Yahia, N. Duval, J.A. de Guise, In vivo reproducibility of a new non-invasive diagnostic tool for three-dimensional knee evaluation, Knee 6 (1999) $175-181$.

[18] N. Hagemeister, G. Parent, M. Van de Putte, N. St-Onge, N. Duval, J. de Guise, A reproducible method for studying three-dimensional knee kinematics, J. Biomech. 38 (2005) 1926-1931.

[19] D.R. Labbe, N. Hagemeister, M. Tremblay, J. de Guise, Reliability of a method for analyzing three-dimensional knee kinematics during gait, Gait Posture 28 (2008) $170-174$.

[20] S. Lustig, R. Magnussen, L. Cheze, P. Neyret, The KneeKG system: a review of the literature, Knee Surg. Sports Traumatol. Arthrosc. 20 (2012) 633-638.

[21] M. Sati, J.A. de Guise, S. Larouche, G. Drouin, Improving in vivo knee kinematic measurements: application to prosthetic ligament analysis, Knee 3 (1996) 179-190.
[22] N. Mannering, T. Young, T. Spelman, P.F. Choong, Three-dimensional knee kinematic analysis during treadmill gait: slow imposed speed versus normal self-selected speed, Bone Joint Res. 6 (2017) 514-521.

[23] E.S. Grood, W.J. Suntay, A joint coordinate system for the clinical description of three-dimensional motions: application to the knee, J. Biomech. Eng. 105 (1983) $136-144$.

[24] M.P. Kadaba, H.K. Ramakrishnan, M.E. Wootten, J. Gainey, G. Gorton, G.V. Cochran, Repeatability of kinematic, kinetic, and electromyographic data in normal adult gait, J. Orthop. Res. 7 (1989) 849-860.

[25] J.J. Cherian, B.H. Kapadia, S. Banerjee, J.J. Jauregui, K. Issa, M.A. Mont, Mechanical, anatomical, and kinematic Axis in TKA: concepts and practical applications, Curr. Rev. Musculoskelet. Med. 7 (2014) 89-95.

[26] M.M. Mukaka, A guide to appropriate use of Correlation coefficient in medical research, Malawi Med. J. 24 (2012) 69-71.

[27] C. Riviere, M. Ollivier, D. Girerd, J.N. Argenson, S. Parratte, Does standing limb alignment after total knee arthroplasty predict dynamic alignment and knee loading during gait? Knee 24 (2017) 627-633.

[28] K.F. Orishimo, I.J. Kremenic, A.J. Deshmukh, S.J. Nicholas, J.A. Rodriguez, Does total knee arthroplasty change frontal plane knee biomechanics during gait? Clin. Orthop. Relat. Res. 470 (2012) 1171-1176.

[29] E.J. Miller, M.W. Pagnano, K.R. Kaufman, Tibiofemoral alignment in posterior stabilized total knee arthroplasty: static alignment does not predict dynamic tibial plateau loading, J. Orthop. Res. 32 (2014) 1068-1074.

[30] W. Blakeney, J. Clément, F. Desmeules, N. Hagemeister, C. Rivière, P.-A. Vendittoli, Kinematic alignment in total knee arthroplasty better reproduces normal gait than mechanical alignment, Knee Surg. Sports Traumatol. Arthrosc. (2018).

[31] J.M. Victor, D. Bassens, J. Bellemans, S. Gursu, A.A. Dhollander, P.C. Verdonk, Constitutional varus does not affect joint line orientation in the coronal plane, Clin. Orthop. Relat. Res. 472 (2014) 98-104.

[32] J. Hutt, V. Massé, M. Lavigne, P.-A. Vendittoli, Functional joint line obliquity after kinematic total knee arthroplasty, Int. Orthop. 40 (2016) 29-34.

[33] H.-M. Ji, J. Han, D.S. Jin, H. Seo, Y.-Y. Won, Kinematically aligned TKA can align knee joint line to horizontal, Knee Surg. Sports Traumatol. Arthrosc. 24 (2016) $2436-2441$. 